\title{
DIFFERENTIABILITY OF DISTANCE FUNCTIONS AND A PROXIMINAL PROPERTY INDUCING CONVEXITY
}

\author{
J. R. GILES
}

(Communicated by William J. Davis)

\begin{abstract}
In a normed linear space $X$, consider a nonempty closed set $K$ which has the property that for some $r>0$ there exists a set of points $x_{0} \in X \backslash K, d\left(x_{0} K\right)>r$, which have closest points $p\left(x_{0}\right) \in K$ and where the set of points $x_{0}-r\left(\left(x_{0}-p\left(x_{0}\right)\right) /\left\|x_{0}-p\left(x_{0}\right)\right\|\right)$ is dense in $X \backslash K$. If the norm has sufficiently strong differentiability properties, then the distance function $d$ generated by $K$ has similar differentiability properties and it follows that, in some spaces, $K$ is convex.
\end{abstract}

Given a real normed linear space $X$, a subset $K$ is called a proximinal (Chebyshev) set if for each $x \in X \backslash K$ there exists a (unique) $p(x) \in K$ such that

$$
\|x-p(x)\|=d(x, K) \equiv d(x) .
$$

It has long been known that in smooth finite-dimensional normed linear spaces every Chebyshev set is convex. In such spaces the metric projection $x \mapsto p(x)$ is continuous on $X \backslash K$ and this fact is used in the proof. So it has been natural to assume the continuity of the metric projection when attempting to prove the convexity of Chebyshev sets in smooth infinite-dimensional spaces. The best result so far has been given by Vlasov $[10,11]$ who showed that in a Banach space with rotund dual, Chebyshev sets which have continuous metric projection are convex. For many years it has been a matter of speculation whether there exist nonconvex Chebyshev sets in Hilbert space [8]; both Vlasov and Asplund [1] showed that in Hilbert space a Chebyshev set with continuous metric projection is convex.

The continuity of the metric projection was shown to be not necessary when a smooth and rotund space isomorphic to Hilbert space was exhibited containing a Chebyshev subspace with discontinuous metric projection [4]. However, an examination of Vlasov's proof $[7$, p. 238] shows that he uses the continuity of the metric projection only to establish a differentiability property of the distance function generated by the set. Moreover stated in terms of a differentiability condition on the distance function, reference to a proximinal condition can be removed. In fact, Vlasov's Theorem can be stated as follows.

Received by the editors May 14, 1987 and, in revised form, August 15, 1987.

1980 Mathematics Subject Classification (1985 Revision). Primary 41A65, 46B20.

Key words and phrases. Distance functions, metric projection, proximinal, Chebyshev sets, Gâteaux, Fréchet, uniformly Gâteaux, uniformly Fréchet differentiable.

The author wishes to express his thanks to Professor Susanna Papadopoulou for providing hospitality at the University of Crete enabling him to work on this research. 
Proposition 1 [2, THEOREMS 14-18]. In a Banach space $X$ with rotund dual $X^{*}$, a nonempty closed set $K$ is convex if its distance function $d$ satisfies

$$
\limsup _{\|y\| \rightarrow 0} \frac{d(x+y)-d(x)}{\|y\|}=1 \quad \text { for all } x \in X \backslash K .
$$

In particular, this differentiability condition is satisfied if $d$ is Gâteaux differentiable and $\left\|d^{\prime}(x)\right\|=1$ or if $d$ is Fréchet differentiable for all $x \in X \backslash K$.

It is easily seen that in any normed linear space $X$, if every point $x \in X \backslash K$ is an interior point of an interval with endpoints $x_{0} \in X \backslash K$ and closest point $p\left(x_{0}\right) \in K$, then $p(x)=p\left(x_{0}\right)$ and the elementary differentiability condition will be satisfied. Further, it is not difficult to see that for the convexity of $K$ in a normed linear space $X$, it is a necessary condition that the distance function $d$ satisfy the elementary diferentiability condition $x \in X \backslash K$. We will use Vlasov's Theorem in the form of Proposition 1 to establish the corollary to our theorem.

In his papers $[\mathbf{5}, \mathbf{6}]$, Fitzpatrick explored the relationship between continuity of the metric projection and differentiability of the distance function, and it has been this investigation which has been an incentive to examine differentiability properties of the distance function and their influence in determining convexity. I am indebted to him for observing that my results hold in a more general setting than I at first claimed.

A real function $\phi$ on a normed linear space $X$ is said to be Gâteaux differentiable at $x \in X$ if there exists a linear functional $\phi^{\prime}(x)$ on $X$ called the Gateaux derivative of $\phi$ at $x$, where, given $\varepsilon>0$ and $y \in X,\|y\|=1$, there exists a $\delta(\varepsilon, x, y)>0$ such that

$$
\left|(\phi(x+t y)-\phi(x)) / t-\phi^{\prime}(x)(y)\right|<\varepsilon \quad \text { when } 0<|t|<\delta .
$$

The function $\phi$ is said to be Fréchet differentiable at $x$ if there exists a $\delta(\varepsilon, x)>0$ such that the inequality holds for all $y \in X,\|y\|=1$. The function $\phi$ is said to be uniformly Gâteaux differentiable on a subset $D$ if there exists a $\delta(\varepsilon, y)>0$ such that the inequality holds for all $x \in D$, and is said to be uniformly Fréchet differentiable on a subset $D$ if there exists a $\delta(\varepsilon)>0$ such that the inequality holds for all $x \in D$ and all $y \in X,\|y\|=1$. When the norm of $X$ is Gâteaux differentiable at $x \neq 0$ we denote the Gâteaux derivative by $f_{x}$ and we note that $\left\|f_{x}\right\|=1$. We say that $X$ has uniformly Gâteaux (uniformly Fréchet) differentiable norm if the norm is uniformly Gâteaux (uniformly Fréchet) differentiable on $\{x:\|x\|=1\}$.

In a normed linear space, uniform Gâteaux differentiability of the norm has remarkable consequences for any distance function. In such a space the distance function $d$ generated by a nonempty closed set $K$ always has a right-hand derivative $d_{+}^{\prime}(x)(y)$ at each $x \in X \backslash K$ and this function is concave in $y[\mathbf{1 2}, \mathrm{p}$. 300]. But further we are able to obtain a particularly useful characterization for Gâteaux differentiability of the distance function. When the norm is uniformly Fréchet differentiable we are able to derive a similar characterisation for Fréchet differentiability of the distance function.

Given a nonempty closed set $K$ in a normed linear space $X$ we denote by $P(K)$ a set of points $x \in X \backslash K$ where there exist points $p(x) \in K$ such that $\|x-p(x)\|=$ $d(x)$. For any $x \in P(K)$ with closest point $p(x) \in K$ we will denote by $\vec{x}$ the unit element in the direction $x-p(x)$. 
PROPOSITION 2. In a normed linear space $X$ with uniformly Gâteaux (uniformly Fréchet) differentiable norm, given a nonempty closed set $K$ with a set $P(K)$ dense in $X \backslash K$, and choosing for each $x \in P(K)$ a closest point $p(x) \in K$, then the distance function d generated by $K$ is Gâteaux (Fréchet) differentiable at $x \in X \backslash K$ if and only if $\left\{f_{\vec{z}_{n}}\right\}$ is weak ${ }^{*}$ convergent (norm convergent) for $z_{n} \in P(K)$ and $\left\{z_{n}\right\}$ converging to $x$. (Of course, if $\left\{f_{\vec{z}_{n}}\right\}$ is weak convergent for $z_{n} \in P(K)$ and $\left\{z_{n}\right\}$ converging to $x$, then it is weak $k^{*}$ convergent to $d^{\prime}(x)$ the Gâteaux derivative of $d$ at $x$.)

PROOF. The proof when the norm is uniformly Gâteaux differentiable is contained in [3, Theorem 4 and 2, Corollary 9]. We need only consider the case when the norm is uniformly Fréchet differentiable.

Suppose that for every sequence $\left\{z_{n}\right\}$ in $P(K)$ where $\left\{z_{n}\right\}$ converges to $x$, the sequence $\left\{f_{\vec{z}_{n}}\right\}$ is norm convergent. Then $d$ is Gâteaux differentiable at $x$. Suppose that $d$ is not Fréchet differentiable at $x$. Then there exists an $r>0$ and a sequence $\left\{y_{n}\right\}$ in $X$ where $y_{n} \rightarrow 0$ such that

$$
r\left\|y_{n}\right\|<d\left(x+y_{n}\right)-d(x)-d^{\prime}(x)\left(y_{n}\right) .
$$

Since $P(K)$ is dense in $X \backslash K$, for each $n$ choose $z_{n} \in P(K)$ such that $\left\|z_{n}-x\right\|<$ $n^{-1}\left\|y_{n}\right\|$. Then

$$
\begin{aligned}
r\left\|y_{n}\right\| & <d\left(z_{n}+y_{n}\right)-d\left(z_{n}\right)-d^{\prime}(x)\left(y_{n}\right)+2 n^{-1}\left\|y_{n}\right\| \\
& <\left\|z_{n}-p\left(z_{n}\right)+y_{n}\right\|-\left\|z_{n}-p\left(z_{n}\right)\right\|-d^{\prime}(x)\left(y_{n}\right)+2 n^{-1}\left\|y_{n}\right\| .
\end{aligned}
$$

Since the norm is uniformly Fréchet differentiable, given $0<\varepsilon<r / 2$ there exists a $\delta(\varepsilon)>0$ such that

$$
\left|\left\|z_{n}-p\left(z_{n}\right)+y_{n}\right\|-\left\|z_{n}-p\left(z_{n}\right)\right\|-f_{\vec{z}_{n}}\left(y_{n}\right)\right|<\varepsilon\left\|y_{n}\right\| \quad \text { for all }\left\|y_{n}\right\|<\delta .
$$

Then

$$
\frac{1}{2} r\left\|y_{n}\right\|<f_{\vec{z}_{n}}\left(y_{n}\right)-d^{\prime}(x)\left(y_{n}\right)+2 n^{-1}\left\|y_{n}\right\| \quad \text { for all }\left\|y_{n}\right\|<\delta
$$

so

$$
\frac{1}{2} r<\left\|f_{\vec{z}_{n}}-d^{\prime}(x)\right\|+2 n^{-1}
$$

and we conclude that $\left\{f_{\vec{z}_{n}}\right\}$ is not norm convergent to $d^{\prime}(x)$ although $\left\{z_{n}\right\}$ converges to $x$.

Suppose that $d$ is Fréchet differentiable at $x \in X \backslash K$. Suppose also that there exists a sequence $\left\{z_{n}\right\}$ in $P(K)$ converging to $x$ but where $\left\{f_{\vec{z}_{n}}\right\}$ is not norm convergent to $d^{\prime}(x)$. Then there exists an $r>0$ and a subsequence of $\left\{z_{n}\right\}$ such that

$$
\left\|f_{\vec{z}_{n}}-d^{\prime}(x)\right\|>3 r \text { for all } n .
$$

So there exists a sequence $\left\{y_{n}\right\}$ in $X,\left\|y_{n}\right\|=1$ such that

$$
-f_{\vec{z}_{n}}\left(y_{n}\right)+d^{\prime}(x)\left(y_{n}\right)>3 r \text { for all } n \text {. }
$$

Since $d$ is Fréchet differentiable at $x$ there exists a $\delta(\varepsilon, x)>0$ such that

$$
\left|d(x+w)-d(x)-d^{\prime}(x)(w)\right|<r\|w\| \text { for all }\|w\| \leq \delta .
$$


Putting $w_{n} \equiv \delta y_{n}$ we have $\left\|w_{n}\right\|=\delta$ for all $n$, and for each $n$,

$$
\begin{aligned}
3 r \delta< & -f_{\vec{z}_{n}}\left(w_{n}\right)+d^{\prime}(x)\left(w_{n}\right) \\
< & d^{\prime}(x)\left(w_{n}\right)-d\left(x+w_{n}\right)+d(x)+d\left(z_{n}+w_{n}\right)-d\left(z_{n}\right)-f_{\vec{z}_{n}}\left(w_{n}\right) \\
& +\left\|z_{n}-x\right\|+d\left(z_{n}\right)-d(x) \\
< & r \delta+\left\|z_{n}-p\left(z_{n}\right)+w_{n}\right\|-\left\|z_{n}-p\left(z_{n}\right)\right\|-f_{\vec{z}_{n}}\left(w_{n}\right) \\
& +\left\|z_{n}-x\right\|+d\left(z_{n}\right)-d(x) .
\end{aligned}
$$

Since the norm is uniformly Fréchet differentiable there exists a $0<\delta^{\prime}<\delta$ such that

$$
\begin{aligned}
\mid\left\|z_{n}-p\left(z_{n}\right)+w_{n}\right\| & -\left\|z_{n}-p\left(z_{n}\right)\right\|-f_{\vec{z}_{n}}\left(w_{n}\right) \mid \\
& <r\left\|w_{n}\right\| \text { for all } z_{n} \text { and }\left\|w_{n}\right\|<\delta^{\prime} \\
& <r \delta
\end{aligned}
$$

So

$$
r \delta<\left\|z_{n}-x\right\|+d\left(z_{n}\right)-d(x) .
$$

But this contradicts the continuity of $d$.

The following lemma, using a construction of Fitzpatrick [6], shows the significance for distance functions of differentiability in closest directions.

LEMMA. If a normed linear space $X$ has uniformly Gâteaux (uniformly Fréchet) differentiable norm and for a nonempty closed set $K, D$ is a subset of $P(K)$ where $d$ is differentiable at each $x \in D$ in the direction of a closest point $p(x) \in K$ uniformly on $D$, then $d$ is uniformly Gâteaux (uniformly Fréchet) differentiable on $D$.

ProOF. Given $\varepsilon>0$ and $y \in X,\|y\|=1$, there exists a $\gamma(\varepsilon, y)>0(\gamma(\varepsilon)>0)$ such that

$$
\left|(\|\vec{x}+\lambda y\|-\|x\|) / \lambda-f_{\vec{x}}(y)\right|<\varepsilon \quad \text { for all } x \in D, 0<|\lambda| \leq \gamma
$$

so

$$
\|\| \vec{x}+\gamma y\|-\| \vec{x} \|-\gamma f_{\vec{x}}(y) \mid<\varepsilon \gamma .
$$

Also there exists a $\delta(\varepsilon)>0$ such that

$$
|(d(x+t \vec{x})-d(x)) / t-1| \leq \varepsilon \gamma \quad \text { for all } x \in D, 0<|t|<\delta .
$$

Then

$$
\begin{aligned}
d(x+t \gamma y)-d(x) & =d(x+t \gamma y)-d(x-t \vec{x})+d(x-t \vec{x})-d(x) \\
& <t(\|\vec{x}+\gamma y\|-\|\vec{x}\|)+\varepsilon \gamma t \quad \text { for } 0<t<\delta \\
& <t \gamma f_{\vec{x}}(y)+2 \varepsilon \gamma t .
\end{aligned}
$$

So

$$
(d(x+t \gamma y)-d(x)) / t \gamma<f_{\vec{x}}(y)+2 \varepsilon \quad \text { for } 0<t<\delta .
$$

On the other hand,

$$
\begin{aligned}
d(x+t \gamma y)-d(x) & =d(x+t \gamma y)-d(x+t \vec{x})+d(x+t \vec{x})-d(x) \\
& >-t(\|\vec{x}-\gamma y\|-\|\vec{x}\|)-\varepsilon \gamma t \quad \text { for } 0<t<\delta \\
& >t \gamma f_{\vec{x}}(y)-2 \varepsilon \gamma t .
\end{aligned}
$$


So

$$
(d(x+t \gamma y)-d(x)) / t \gamma>f_{\vec{x}}(y)-2 \varepsilon \text { for } 0<t<\delta .
$$

From (i) and (ii) we conclude that $d$ is uniformly Gâteaux (uniformly Fréchet) differentiable on $D$.

Given a nonempty closed set $K$ in a normed linear space $X$ and $r>0$, we denote by $P_{r}(K)$ the set of points $x_{0}-r \vec{x}_{0}$ where $x_{0} \in P(K), p\left(x_{0}\right) \in K$ and $\left\|x_{0}-p\left(x_{0}\right)\right\|=d\left(x_{0}, K\right)>r$.

We are now ready to present our theorem.

THEOREM. In a normed linear space $X$ with uniformly Gâteaux (uniformly Fréchet) differentiable norm, a nonempty closed set $K$ generates a Gâteaux (Fréchet) differentiable distance function $d$ on $X \backslash K$ if for some $P(K)$ and $r>0$, the set $P_{r}(K)$ is dense in $X \backslash K$.

Proof. Consider $\bar{x} \in X \backslash K$ and $\bar{r}>0$ such that $d(B(\bar{x} ; \bar{r}), K)>0$. Then for every $v \in P_{r}(K) \cap B(\bar{x} ; \bar{r})$,

$$
d(v+t \vec{v})=\|v-p(v)\|+t
$$

for $0<|t|<\min (r, d(B(\bar{x} ; \bar{r}), K))$. So $d$ is differentiable in the direction of its closest points uniformly on $P_{r}(K) \cap B(\bar{x} ; \bar{r})$. It now follows from the Lemma that $d$ is uniformly Gâteaux (uniformly Fréchet) differentiable on $P_{r}(K) \cap B(\bar{x} ; \bar{r})$. Therefore, given $\varepsilon>0$ and $y \in X,\|y\|=1$, there exists a $\delta(\varepsilon, y)>0(\delta(\varepsilon)>0)$ such that

$$
\left|(d(x+t y)-d(x)) / t-f_{\vec{x}}(y)\right|<\varepsilon \quad \text { for all } x \in P_{r}(K) \cap B(\bar{x}, \bar{r}), 0<|t|<\delta .
$$

Then for $x, z \in P_{r}(K) \cap B(\bar{x} ; \bar{r})$ and $y \in X,\|y\|=1$,

$$
\begin{aligned}
\mid\left(f_{\vec{x}}-f_{\vec{z}}(y) \mid \leq\right. & \left|f_{\vec{x}}(y)-\frac{d(x+t y)-d(x)}{t}\right| \\
& +\left|\frac{d(x+t y)-d(x)}{t}-\frac{d(z+t y)-d(z)}{t}\right| \\
& +\left|f_{\vec{z}}(y)-\frac{d(z+t y)-d(z)}{t}\right| \\
< & 2 \varepsilon+\| x-z|| 4 / \delta \text { for all } \delta / 2<|t|<\delta \\
< & 6 \varepsilon \text { for all }\|x-z\|<\varepsilon \delta .
\end{aligned}
$$

That is, the mapping $x \mapsto f_{\vec{x}}(y)\left(x \mapsto f_{\vec{x}}\right)$ is uniformly continuous on $P_{r}(K) \cap$ $B(\bar{x} ; \bar{r})$. Since $P_{r}(K)$ is dense in $X \backslash K$, this mapping has a unique continuous extension on $B(\bar{x} ; \bar{r})$. But this implies that for any $x \in B(\bar{x} ; \bar{r})$ and sequence $\left\{z_{n}\right\}$ in $P_{r}(K) \cap B(\bar{x} ; \bar{r})$ converging to $x,\left\{f_{\vec{z}_{n}}\right\}$ is weak* convergent (norm convergent). It then follows from Proposition 2 that $d$ is Gâteaux (Fréchet) differentiable at $x$.

Using Proposition 1 we can give the following conditions for the convexity of a set. 
COROLlARY. In a Banach space $X$, consider a nonempty closed set $K$ with the property that for some $P(K)$ and $r>0$ the set $P_{r}(K)$ is dense in $X \backslash K$. If

(i) $X$ has uniformly Gateaux differentiable norm and the distance function $d$ has $\left\|d^{\prime}(x)\right\|=1$ for all $x \in X \backslash K$, or

(ii) $X$ has uniformly Fréchet differentiable norm, then $K$ is convex.

We note that any proximinal set $K$ with distance function $d$ Gâteaux differentiable on $X \backslash K$ has $\left\|d^{\prime}(x)\right\|=1$ for all $x \in X \backslash K$. So if $X$ has uniformly Gâteaux differentiable norm and $K$ is proximinal and satisfies the proximinal condition, then $K$ is convex.

In a rotund normed linear space $X$ with uniformly Gâteaux differentiable norm, if $K$ satisfies the proximinal condition then every point $x \in X \backslash K$ which has a closest point in $K$ has a unique closest point in $K$. For suppose that there exists a point $x \in X \backslash K$ with two closest points $p_{1}(x), p_{2}(x) \in K$ and denote by $\vec{x}_{1}$ and $\vec{x}_{2}$ the unit vectors in the direction $x-p_{1}(x)$ and $x-p_{2}(x)$. From the Theorem, $d$ is Gâteaux differentiable at $x$ and $d^{\prime}(x)=f_{\vec{x}_{1}}=f_{\vec{x}_{2}}$. Since $X$ is rotund we conclude that $p_{1}(x)=p_{2}(x)$. Conversely, if the Chebyshev property implied the proximinal condition then in Hilbert space every Chebyshev set would be convex.

Lau $[9$, p. 794] has shown that in any reflexive Banach space $X$ with Kadec norm, every nonempty closed set $K$ has a set $P(K)$ dense in $X \backslash K$; in particular every Hilbert space has this property. We note that if there exists an $r>0$ and a set $P_{r}(K)$ dense in $X \backslash K$ then there exists a set $P(K)$ which is dense in $X \backslash K$; if the converse were true then in Hilbert space every Chebyshev set would be convex.

A proximinal set $K$ in a normed linear space $X$ is a sun if for every $x \in X \backslash K$ with a closest point $p(x) \in K$, points $x+t \vec{x}$ have $p(x)$ as a closest point for all $t \geq 0$. If for a proximinal set $K$ and some $r>0, P_{r}(K)$ is not dense in $X \backslash K$, then there exists an $x_{0} \in X \backslash K$ and an $0<r^{\prime}<r$ such that $B\left(x_{0} ; r^{\prime}\right) \cap P_{r}(K)=\varnothing$. Then $p\left(x_{0}\right)$ is not a closest point for $x_{0}+t \vec{x}_{0}$ when $r-r^{\prime}<t<r+r^{\prime}$ and so $K$ is not a sun. As a convex proximinal set is a sun, we conclude that the proximinal condition in our Theorem is a necessary condition for the convexity of a Chebyshev set and so, in a Banach space with rotund dual, such a condition is less restrictive than the continuity of the metric projection.

Finally, it should be noted that if there does exist a nonconvex Chebyshev set $K$ in a Hilbert space $X$, then even though we have $P(K)=X \backslash K$, for each $r>0$ there exists a "black hole" open ball which contains no points of $P_{r}(K)$.

NOTE ADDED IN PROOF. It is not difficult to show directly that the proximinal condition implies the Vlasov differentiability condition and so the following result holds which is more general than the above Corollary.

THEOREM. In a Banach space $X$ with rotund dual $X^{*}$ a nonempty closed set $K$ is convex if for some $P(K)$ and $r>0$ the set $P_{r}(K)$ is dense in $X \backslash K$.

ProOF. For $x \in X \backslash K$ there exists a sequence $\left\{x_{n}\right\}$ in $P_{r}(K) \backslash\{x\}$ converging to $x$. Then given $0<\varepsilon<r$, for $x_{n_{0}} \equiv x_{n}+\varepsilon \vec{x}_{n}$,

$$
\begin{aligned}
d\left(x_{n_{0}}\right)-d(x) & =d\left(x_{n}\right)+\left\|x_{n_{0}}-x_{n}\right\|-d(x) \\
& \geq\left\|x_{n_{0}}-x\right\|-2\left\|x_{n}-x\right\|
\end{aligned}
$$


and

$$
\frac{d\left(x_{n_{0}}\right)-d(x)}{\left\|x_{n_{0}}-x\right\|} \geq 1-\frac{2\left\|x_{n}-x\right\|}{\varepsilon-\left\|x_{n}-x\right\|} .
$$

We deduce that

$$
\limsup _{\|y\| \rightarrow 0} \frac{d(x+y)-d(x)}{\|y\|}=1 \text {. }
$$

\section{REFERENCES}

1. E. Asplund, Chebyshev sets in Hilbert space, Trans. Amer. Math. Soc. 144 (1969), 235-240.

2. J. M. Borwein, S. P. Fitzpatrick and J. R. Giles, The differentiability of real functions on normed linear spaces using generalized subgradients, J. Math. Anal. Appl. 128 (1987), 512-534.

3. J. M. Borwein and J. R. Giles, The proximal normal formula in Banach space, Trans. Amer. Math. Soc. 302 (1987), 371-381.

4. A. L. Brown, A rotund, reflexive space having a subspace of co-dimension two with discontinuous metric projection, Michigan Math. J. 21 (1974), 145-151.

5. Simon Fitzpatrick, Metric projections and the differentiability of distance functions, Bull. Austral. Math. Soc. 22 (1980), 291-312.

6. _ Differentiation of real-valued functions and continuity of metric projections, Proc. Amer. Math. Soc. 91 (1984), 544-548.

7. J. R. Giles, Convex analysis with application in the differentiation of convex functions, Research Notes in Math., 58, Pitman, 1982.

8. V. L. Klee, Dispersed Chebyshev sets and coverings by balls, Math. Ann. 257 (1981), 251-260.

9. K. S. Lau, Almost Chebyshev subsets in reflexive Banach spaces, Indiana Univ. Math. J. 2 (1978), 791-795.

10. L. P. Vlasov, Chebyshev sets and approximatively convex sets, Math. Notes Acad. Sci. USSR 2 (1967), 600-605.

11. __ Almost convex and Chebyshev sets, Math. Notes Acad. Sci. USSR 8 (1970), 776-779.

12. L. Zajícek, Differentiability of the distance function and points of multivaluedness of the metric projection in Banach spaces, Czechoslovak Math. J. 33 (1983), 292-308.

Department of Mathematics, University of Newcastle, New South Wales, Australia 2308 